\title{
Detection of Burkholderia pseudomallei O-antigen serotypes in near-neighbor species
}

\author{
Joshua K Stone, Mark Mayo², Stephanie A Grasso', Jennifer L Ginther', Stephanie D Warrington', \\ Christopher J Allender ${ }^{1}$, Adina Doyle ${ }^{1}$, Shalamar Georgia', Mirjam Kaestli², Stacey M Broomall ${ }^{3}$, Mark A Karavis ${ }^{3}$, \\ Joseph M Insalaco ${ }^{3}$, Kyle S Hubbard ${ }^{3}$, Lauren A McNew ${ }^{3}$, Henry S Gibbons ${ }^{3}$, Bart J Currie ${ }^{2}$, Paul Keim ${ }^{1}$ and \\ Apichai Tuanyok ${ }^{* *}$
}

\begin{abstract}
Background: Burkholderia pseudomallei is the etiological agent of melioidosis and a CDC category B select agent with no available effective vaccine. Previous immunizations in mice have utilized the lipopolysaccharide (LPS) as a potential vaccine target because it is known as one of the most important antigenic epitopes in B. pseudomallei. Complicating this strategy are the four different B. pseudomallei LPS O-antigen types: A, B, B2, and rough. Sero-crossreactivity is common among O-antigens of Burkholderia species. Here, we identified the presence of multiple B. pseudomallei O-antigen types and sero-crossreactivity in its near-neighbor species.

Results: PCR screening of O-antigen biosynthesis genes, phenotypic characterization using SDS-PAGE, and immunoblot analysis showed that majority of $B$. mallei and B. thailandensis strains contained the typical O-antigen type A. In contrast, most of B. ubonensis and B. thailandensis-like strains expressed the atypical O-antigen types B and $B 2$, respectively. Most $B$. oklahomensis strains expressed a distinct and non-seroreactive O-antigen type, except strain E0147 which expressed O-antigen type A. O-antigen type B2 was also detected in B. thailandensis 82172, B. ubonensis MSMB108, and Burkholderia sp. MSMB175. Interestingly, B. thailandensis-like MSMB43 contained a novel serotype B positive O-antigen.

Conclusions: This study expands the number of species which express B. pseudomallei O-antigen types. Further work is required to elucidate the full structures and how closely these are to the B. pseudomallei O-antigens, which will ultimately determine the efficacy of the near-neighbor B serotypes for vaccine development.
\end{abstract}

\section{Background}

Lipopolysaccharide (LPS) is an amphiphilic molecule which is a major component in the outer membrane of Gram-negative bacteria [1]. It is composed of three parts - a membrane bound lipid A, or endotoxin, a core oligosaccharide, and a repeating $\mathrm{O}$-antigen [2]. The lipid $\mathrm{A}$ is the signal that triggers the innate immune system during infection and is structurally conserved across genera with differences in immune response attributable to the presence of varying fatty acids $[1,3,4]$. The $\mathrm{O}$-antigen is the most structurally diverse LPS component within a species, with over 170 known structures in Escherichia coli

\footnotetext{
* Correspondence: Apichai.Tuanyok@nau.edu

${ }^{1}$ Center for Microbial Genetics and Genomics, Northern Arizona University, Flagstaff, AZ, USA

Full list of author information is available at the end of the article
}

alone [1]. As an antigenic determinant, $\mathrm{O}$-antigen structures can be grouped by serotype [2].

Burkholderia pseudomallei is a saprophytic Gramnegative bacterium endemic to Southeast Asia and Australia. It is the etiological agent of the septicemic disease melioidosis and a CDC category $B$ select agent with no available effective vaccine [5,6]. However, limited success has been met with use of LPS from $B$. pseudomallei and the avirulent near-neighbor $B$. thailandensis in rodent and rabbit melioidosis models [7-10]. Four distinct O-antigen ladder patterns have been described in B. pseudomallei, known as types A, $B, B 2$, and rough, which lacks the repeating unit [11]. Most B. pseudomallei strains express type A O-antigen, making it by far the most abundant structure, whereas the atypical types, B and B2, are serologically related but have distinct ladder banding patterns when run on

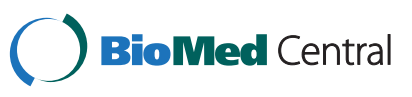


SDS-PAGE [11]. Type A is also found in B. thailandensis and the virulent $B$. mallei $[12,13]$. This is also the only $\mathrm{O}$-antigen that has been structurally characterized, containing a disaccharide 3)- $\beta$-D-glucopyranose-(1,3)$6 \mathrm{~d}-\alpha$-L-talopyranose-( 1 repeat, with the talose residue variably acetylated and methylated [13-16]. Type B has not been found in any other species while type B2 was recently described in a $B$. thailandensis-like species [11]. B. thailandensis-like species is a new species within the Pseudomallei phylogenetic group which is closely related to $B$. pseudomallei and $B$. thailandensis. This new species was first discovered in soil and water in northern Australia [17]. The presence of types A and B2 in near-neighbor species suggests that further screening will reveal additional species expressing $B$. pseudomallei $\mathrm{O}$-antigen types.

In our present study, LPS genotyping and phenotypic analyses of numerous near-neighbor isolates suggested the presence of type $\mathrm{A}$ in $B$. mallei, B. thailandensis, and B. oklahomensis; type B in B. ubonensis; and type B2 in $B$. thailandensis, a $B$. thailandensis-like species, and $B$. ubonensis. Representative strains containing B. pseudomallei $\mathrm{O}$-antigen ladder banding patterns were chosen for further whole genome sequencing and subjected to comparative genomics.

\section{Results}

\section{S rRNA and recA sequencing}

We confirmed bacterial species on all 113 bacterial strains using $16 \mathrm{~S}$ rRNA and $r e c A$ sequencing techniques compared to reference strains available in GenBank. Cutoffs of $99 \%$ and $94 \%$ were established for species classification for $16 \mathrm{~S}$ and $r e c A$ analyses, respectively (data not shown). We identified 23 B. mallei, 4 B. oklahomensis, $12 B$. thailandensis, 5 B. thailandensis-like species, 44 B. ubonensis, and 25 unidentified Burkholderia species strains.

\section{LPS genotyping (PCR)}

Eleven out of $12 \mathrm{~B}$. thailandensis strains had the LPS genotype A. All 23 tested $B$. mallei strains also had the LPS genotype A. LPS genotype B was detected in 11 out of 44 strains of B. ubonensis. We note that these LPS genotype $B$ strains were all of Australian origin. LPS genotype B2 was found in B. thailandensis strain 82172, and B. thailandensis-like species strains MSMB121, MSMB122, MSMB712, and MSMB714. This is the first reported incidence of another O-antigen in B. thailandensis while $B$. thailandensis-like MSMB121 was previously described as expressing this type [11]. No other species was positive for type A, B, or B2 (Table 1 and Additional file 1: Table S1).

\section{LPS phenotyping (SDS-PAGE, silver staining and immunoblotting)}

We identified LPS banding patterns in all tested bacterial strains by comparing them with known LPS banding patterns $\mathrm{A}, \mathrm{B}$, and $\mathrm{B} 2$ in reference $\mathrm{B}$. pseudomallei strains (Additional file 2: Figure S1). Previously, only type A O-antigen has been described in B. thailandensis $[11,12]$. Eleven out of 12 tested strains expressed a type A banding pattern consistent with the PCR results. We note that $B$. thailandensis strain 82172 had the LPS genotype B2 via PCR, which was confirmed as serotype B by immunoblotting (Figure 1). B. pseudomallei strains expressing type B2 have previously been isolated only in Australia and Papua New Guinea, while this B. thailandensis strain was isolated in France $[11,18]$. Additionally, type A was recently described in B. oklahomensis E0147 [11], whereas the remaining three $B$. oklahomensis strains isolated from Oklahoma [19] displayed an unknown non-seroreactive ladder pattern (not shown in Figure 1).

Twenty-one strains of B. mallei expressed type A Oantigen while the remaining two strains (ATCC10399

Table 1 Prevalence of four B. pseudomallei O-antigen types in near-neighbors

\begin{tabular}{|c|c|c|c|c|c|}
\hline \multirow[t]{2}{*}{ Species } & \multirow[t]{2}{*}{ Total strains tested } & \multicolumn{4}{|c|}{ Known B. pseudomallei O-antigen } \\
\hline & & Type A & Type B & Type B2 & Rough Type \\
\hline B. mallei & 23 & 21 & 0 & 0 & 2 \\
\hline B. oklahomensis & 4 & 1 & 0 & 0 & 0 \\
\hline B. thailandensis & 12 & 11 & 0 & $1+$ & 0 \\
\hline B. thailandensis-like & 5 & 0 & 0 & 4 & 0 \\
\hline B. cepacia & 2 & 0 & 0 & 0 & 0 \\
\hline B. multivorans & 3 & 0 & 0 & 0 & 0 \\
\hline B. ubonensis & 44 & 0 & 11 & $1 \neq$ & 0 \\
\hline B. vietnamiensis & 1 & 0 & 0 & 0 & 0 \\
\hline Unidentified Burkholderia spp. & 19 & 0 & 0 & $1^{*}$ & 0 \\
\hline
\end{tabular}

†Strain 82172, collected from French foal.

¥Strain MSMB108, collected from Northern Australian environment

*Strain MSMB175, a soil strain collected from Australia. This strain is currently being proposed as a new Burkholderia species. 


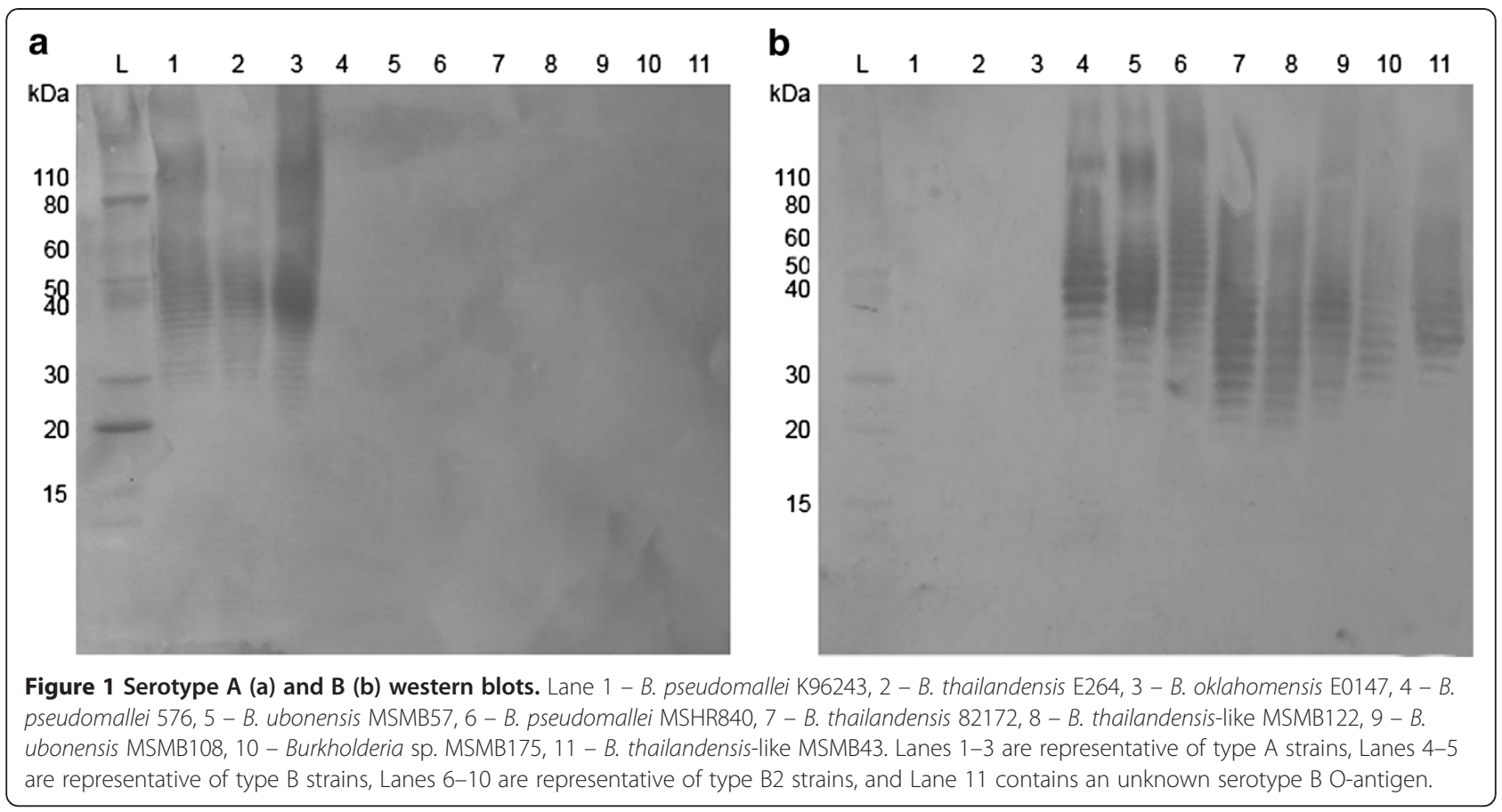

and NCTC120) expressed rough type. ATCC10399 was previously described as having an intact ladder [13,20], but the whole genome sequence (WGS) available in GenBank shows an IS407A insertion in wbiG (NZ_CH899681), which would predict a rough type. IS407A is known as one of the most common insertion sequence (IS) elements in B. pseudomallei and B. mallei [21]. NCTC120's rough type phenotype is consistent with prior works $[13,20]$. Further immunoblotting with the B. mallei LPS-specific mAb 3D11 showed all $21 B$. mallei strains with intact ladder profiles bound this antibody while the two rough type strains did not. B. pseudomallei $\mathrm{K} 96243$ and B. oklahomensis E0147 bound mAb 3D11, as previously described [11]. Similarly, eight of the B. thailandensis strains bound mAb 3D11 while E264, MSMB59, MSMB60, and 82172 did not (Additional file 1: Table S1). Similarly, testing the strains containing type A with the IgM mAb Pp-PS-W, the $B$. pseudomallei LPS-specific mAb [13], showed that $B$. mallei ATCC23344 and B. oklahomensis E0147 were not seroreactive while B. pseudomallei K96243 was seroreactive. Notably, nine $B$. thailandensis strains were seroreactive to this mAb, while MSMB59 and MSMB60 were not. This suggested the existence of seroreactivity diversity within $B$. thailandensis.

PCR suggested that 11 strains of B. ubonensis would be positive for type $\mathrm{B}$ O-antigen. Immunoblotting confirmed the expression of type B in all of these, one of which, MSMB57, was selected for genomic analysis. Another strain, B. ubonensis MSMB108, was negative for all genotypes by PCR but displays a ladder pattern identical to the type B2 B. thailandensis-like MSMB122 (Figure 1). We also noted that other tested $B$. ubonensis strains produced distinct LPS ladder patterns to those of $B$. pseudomallei, which were not seroreactive (data not shown). Along with $B$. thailandensis, $B$. ubonensis was the only species that expressed more than one type of $B$. pseudomallei $\mathrm{O}$-antigen.

B. thailandensis-like strains expressed two different $\mathrm{O}$ antigen ladder patterns, both of which were B serotypes. Strains MSMB121, 122, 712, and 714 expressed ladder type B2 (Additional file 1: Table S1), whereas strain MSMB43 expressed a novel serologically related $\mathrm{O}$-antigen not found in B. pseudomallei. This O-antigen, like type $\mathrm{B} 2$, bound the type $B$ patient's serum but exhibited a banding pattern unlike either type B or B2 (Figure 1). This is the first description of a seroreactive $\mathrm{O}$-antigen found in a near-neighbor species which is unknown in B. pseudomallei.

Burkholderia sp. MSMB175 was negative for all $B$. pseudomallei $\mathrm{O}$-antigen types by PCR. The immunoblotting analysis revealed a banding pattern that was similar to type B2 in higher molecular weight bands (Figure 1). The O-antigen biosynthesis gene cluster for this strain was subsequently sequenced and found to be type B2 (GenBank: JQ783347), with a nucleotide identity of $88 \%$ compared to B. pseudomallei MSHR840.

\section{Genomic analysis}

Genomic comparison has shown that a homolog of wbiE gene in B. oklahomensis E0147 (BoklE_010100014785) had one and five single nucleotide polymorphisms (SNPs) at the forward and reverse primer binding sites, respectively. This caused negative PCR results when the 
previously published LPS genotype A primers [11] were used. In this study, we have adjusted the LPS genotype A primers to be able to amplify all Burkholderia species that contains the LPS genotype A. Similarly, in the type B2 positive Burkholderia sp. MSMB175, two and five SNPs were found in the forward and reverse primer pair binding sites, respectively, revealing why this strain was negative to PCR. In this study, we did not adjust the PCR primers to amplify the LPS genotype B2 in this uncharacterized Burkholderia species.

B. thailandensis E264, MSMB59, and MSMB60 were compared to determine the reason for the differences in sero-reactivity with the mAb Pp-PS-W. Four SNPs were found across the entire gene cluster, however all were synonymous and the amino acid sequences identical (data not shown). In addition, comparison of oacA, the 4- $O$ acetyltransferase gene, sequences also revealed no differences. Further work is required to explain why the Australian isolates fail to cross react with this mAb.

Ten Burkholderia strains were selected for whole genome sequencing to confirm the LPS genotypes. These included B. mallei India 86-567-2, KC237, NCTC120; B. thailandensis MSMB59, MSMB60, 82172; B. thailandensis-like sp. MSMB121, MSMB122; B. ubonensis MSMB57; and Burkholderia sp. MSMB175. Comparative genomics has demonstrated that $\mathrm{O}$-antigen biosynthesis genes in all three sequenced $B$. mallei strains were very similar to those found in a reference LPS genotype A B. mallei ATCC23344, except that strain NCTC120 had an insertion mutation in its wbiE gene (GenBank: JN581992). We noted that the mutation defects the production of Oantigen ladder pattern in this strain (Additional file 1: Table S1). In addition, genomic analysis has shown that $\mathrm{O}-$ antigen genes in B. thailandensis MSMB59 and MSMB60 were very similar to those found in a reference LPS genotype A B. thailandensis E264. Interestingly, B. thailandensis 82172, and B. thailandensis-like sp. strains MSMB121, MSMB122, and Burkholderia sp. MSMB175 had Oantigen genes similar to those found in a reference type B2 B. pseudomallei MSHR840, while B. ubonensis MSMB57 had O-antigen genes which were similar to the genes found in a reference type B B. pseudomallei 576 [11].

One strain of each species expressing $\mathrm{O}$-antigen types $\mathrm{A}, \mathrm{B}$, or B2 were selected for further genomic comparisons. The type A strains B. pseudomallei K96243, B. mallei ATCC23344, B. thailandensis E264, and B. oklahomensis E0147 had an overall nucleotide similarity of 87.2\% to each other, a genic similarity of $87.2 \%$, and an amino acid similarity of $88.7 \%$ (Additional file 3: Figure S2). The type B strains B. pseudomallei 576 and B. ubonensis MSMB57 had an overall nucleotide similarity of $95 \%$, a genic similarity of $95 \%$, and an amino acid similarity of 95\%. The type B2 strains B. pseudomallei MSHR840, B. thailandensis 82172, B. thailandensis-like MSMB122, and
Burkholderia sp. MSMB175 had an overall nucleotide similarity of $90.2 \%$, a genic similarity of $88 \%$, and an amino acid similarity of $86.5 \%$. The diversity of genes that are predicted to be involved in the biosynthesis of LPS types B and B2 is demonstrated in Figure 2. Comparison of the novel B serotype found in B. thailandensis-like MSMB43 with types B and B2 revealed a conservation of the putative epimerase wbil and rhamnose synthesis genes $r m l C A B$ (Figure 2) [11,22]. Transport genes, e.g., $\mathrm{ABC}$-transporters, encoding two wzt and one wzm homologs, are conserved across all three serotype B ladder types. These $w z t$ and $w z m$ homologs are genes BUC_3406, BUC_3409, BURP840_LPSb09, BURP840_LPS12, Bpse38_010100014045, Bpse38_010100014055, and genes BUC_3408, BURP840_LPSb11, Bpse38_010100014050, respectively (Figure 2). These gene products are likely responsible for the sero-crossreactivity observed between these O-antigens (Figure 1). However, a glycosyl transferase gene, Bpse_38010100014060 in B. thailandensis-like MSMB43, which is similar to those found in type B ladder (gene BUC_3410 in B. pseudomallei 576 and gene BuMSMB57_LPSb07 in B. ubonensis MSMB57) has no homology to any of those in the type B2. The type A strains displayed the greatest level of nucleotide diversity, suggesting an ancient acquisition of the gene cluster and a possible ancestral state. Conversely, the type B strains were the most monomorphic, albeit with fewer species representative of this type. In addition, the average $\mathrm{G}+\mathrm{C}$ content of each cluster was $60.8 \%$ for type A, $61 \%$ for type $\mathrm{B}$, and $63.5 \%$ for type B2. Given an average genomic $\mathrm{G}+\mathrm{C}$ content of $68.1 \%$ for the Pseudomallei group, the observed $\mathrm{G}+\mathrm{C}$ content of the $\mathrm{O}$-antigen gene clusters is evidence for horizontal acquisition. This would suggest, however, that type A was unlikely the ancestral type despite being the most abundant and genetically diverse today.

\section{Serum sensitivity}

Previous studies have shown that B. pseudomallei strains with type $\mathrm{B} 2$ or rough type $\mathrm{O}$-antigens display an increased sensitivity to killing by $30 \%$ NHS [11,23]. To determine if near-neighbors showed the same effect, eleven diverse Burkholderia strains expressing type A, B, or B2 O-antigen were assayed for serum sensitivity. All type A strains, B. thailandensis E264, MSMB59, MSMB60, and B. oklahomensis E0147 showed a slight resistance to serum killing, except $B$. thailandensis TXDOH which was sensitive to serum killing. The type B2 B. thailandensis 82172 showed almost no difference in growth, and all other strains were sensitive to killing by $30 \%$ NHS, most notably B. ubonensis MSMB108 (Figure 3).

\section{Discussion}

$\mathrm{O}$-antigen type A has been described as a disaccharide glucose-talose repeat in B. pseudomallei, B. mallei, and 

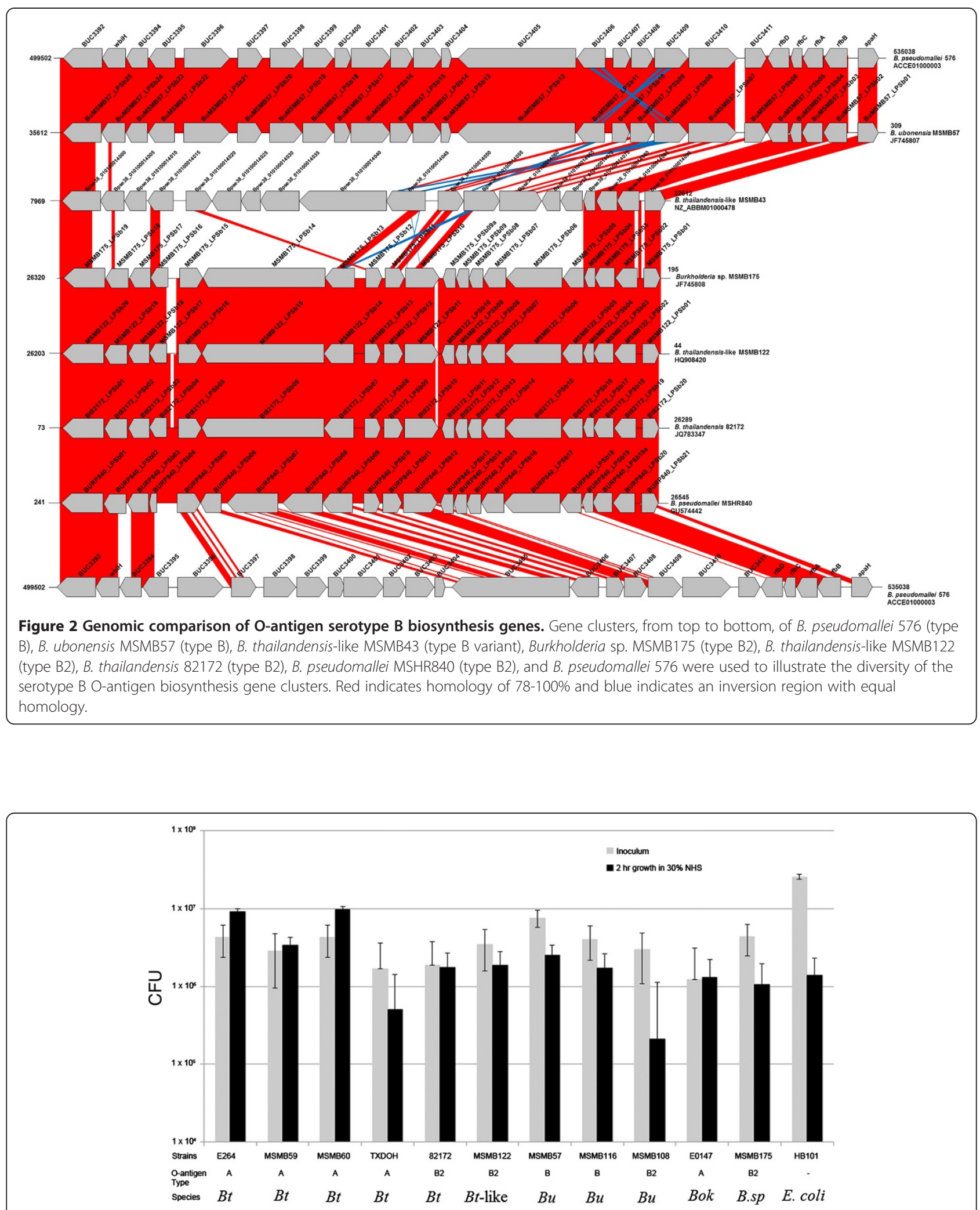

Figure 3 Serum sensitivity of $B$. pseudomallei near-neighbors. B. thailandensis E264, MSMB59, MSMB60 and B. oklahomensis E0147 showed a slight resistance to killing by $30 \%$ NHS while all other strains were susceptible to killing, especially B. ubonensis MSMB108. This is in agreement with prior studies showing serum sensitivity of $B$. pseudomallei strains expressing type B2 or rough type O-antigens. Note: Bt, B. thailandensis; Bt-like, B. thailandensis-like species; Bu, B. ubonensis; Bok, B. oklahomensis; and B.sp, Burkholderia sp. 
B. thailandensis and these structures differ only by side group modification. B. pseudomallei modifies the talose residue with a 2-O methyl/4- $O$ acetyl group or with a 2$\mathrm{O}$ acetyl/4-O hydroxyl group $[15,16]$. In $B$. mallei, regardless of whether the $2-O$ position is methylated or acetylated, the 4-O position remains in its native hydroxyl state [13]. B. thailandensis has been reported to have the same modification patterns as B. pseudomallei $[12,14,22]$, but a recent study by Ngugi, et al., [10] suggests that $B$. thailandensis E264 features a different pattern. Utilizing gas chromatography/mass spectrometry (GC/MS) to examine methylation patterns, they concluded this strain does not methylate the 2-O position. Brett, et al., [14] generated mutants of oacA, the 4-O acetyltransferase gene, which also had the unexpected result of a lack of methylation at the 2-O position. This suggests that the methyl group may be lost during GC/ MS or the E264 strain utilized by Ngugi, et al., [10] may have undergone mutation in oacA, losing its methylase capabilities.

In our current study, 21 out of 23 B. mallei strains expressed intact type $\mathrm{A} \mathrm{O}$-antigens while the remaining two (ATCC10399 and NCTC120) were rough. Two previous studies showed that B. mallei ATCC10399 had a full ladder pattern by silver staining and immunoblotting $[13,20]$. Our genomic analysis has shown that wbiG gene which is known to be involved in the biosynthesis of the type A O-antigen, was disrupted in this strain by IS407A. This supported our finding that ATCC10399 produced rough LPS. B. mallei NCTC120 was also known as a rough LPS type due to the disruption of its $w b i E$, the glycosyltransferase gene, by IS407A [13,20]. DNA sequencing of this strain in our current study revealed the absence of this insertion element, however, a 22 base pair artifact remains in the $3^{\prime}$ end of this gene (GenBank: JN581992), suggesting, IS407A remains active in this strain. We believe that the artifact sequence of the IS407A is disruptive enough to yield the same phenotype as the full insertion.

Eleven strains of B. ubonensis, all Australian environmental isolates, were found to express type $\mathrm{B}$. This $\mathrm{O}-$ antigen type is present in approximately $14 \%$ of all $B$. pseudomallei isolates of which the vast majority are Australian [11]. We report here the first discovery of $B$. pseudomallei type $\mathrm{B} \mathrm{O}$-antigen in a near-neighbor species. Previously, B. ubonenesis was known in Australia from only two strains, only one of which has been sequenced and contains an unknown O-antigen biosynthesis gene cluster (NZ_ABBE01000374) [24]. Environmental sampling in northern Australia yielded 44 total B. ubonensis strains, which was the species most commonly isolated. Conversely, only two B. thailandensis strains were isolated, the same number as Levy, et al., found [24]. While no study has examined the abundance of B. ubonensis in Southeast Asia, it is possible that these two species occupy a similar environmental niche where B. ubonensis is able to outcompete B. thailandensis in Australia. In support this, B. ubonensis isolated from Papua New Guinea exhibited antibiosis against B. pseudomallei [25]. These Australian isolates may produce a similar compound against $B$. thailandensis.

$B$. thailandensis-like species, a new member of the Pseudomallei group, expresses type B2 and a novel ladder pattern seropositive for type $B$, thus far unknown in any other species or strain. Curiously, B. thailandensis 82172 expresses type B2, as well, marking the first description of another $\mathrm{O}$-antigen type in this species. This strain belongs to a distinct phylogenetic cluster along with four other geographically diverse B. thailandensis strains, only one of which was isolated in Asia. This cluster has been suggested as the beginning of a possible speciation event and the discovery of type B2 LPS lends further credence to this idea [26].

Burkholderia sp. MSMB175 is another Australian environmental isolate which clusters with the Pseudomallei group on the basis of $\operatorname{rec} A$ and $16 \mathrm{~S}$ sequence and may represent a new species (data not shown). The presence of type B2 O-antigen (Table 1) supports the possibility that this strain belongs to the Pseudomallei group.

A 1993 study of northeastern Thai children by Kanaphun, et al., [27] revealed that $80 \%$ are seropositive for antibodies against $B$. pseudomallei by the age of four. Accordingly, over 25\% of environmental Burkholderia isolates in Thailand are B. thailandensis [28]. This suggests that some of these children have instead been exposed to this species and not B. pseudomallei, especially given the noted inaccuracies and high background of indirect hemagglutination assays [29]. Little work has examined the seropositive rates in Australia, but two studies in Northern Queensland returned rates of 2.5$5.7 \%$ [30,31]. The high clinical relevance of B. pseudomallei expressing type $\mathrm{B}$ or $\mathrm{B} 2 \mathrm{O}$-antigen, along with the new apparent abundance of these types in Australian near-neighbors, suggest similar exposures may result in false positive diagnoses, as is likely the case in Thailand.

These near-neighbor species are avirulent, B. mallei excepted, and as such are not limited to the biosafety regulations that $B$. pseudomallei is as a biosafety level 3 (BSL-3) organism. Few laboratories worldwide are properly equipped to handle BSL-3 work and so the finding of $B$. pseudomallei type LPS in these non-pathogenic Burkholderia species will allow many additional laboratories the opportunity to work towards vaccine development for melioidosis.

\section{Conclusions}

B. thailandensis type A O-antigen has been used with some success to vaccinate mice against $B$. pseudomallei 
[7-10]. This O-antigen is indistinguishable between these two species in backbone and side group modifications $[12,16,22]$. Given the high genetic similarity between types B and B2 in near-neighbors and B. pseudomallei, it is likely at least one species will be identical in backbone and side group modifications, as well. In such a case, it is possible that particular strain or strains will confer comparable host immunity upon subsequent challenge with type B or B2 B. pseudomallei in much the same way B. thailandensis protects against type A B. pseudomallei challenge.

\section{Methods}

\section{Bacterial strains, DNA, and LPS preparations}

A total of 113 strains of $B$. pseudomallei near-neighbors were used in this study. These included 23 B. mallei, 4 B. oklahomensis, 12 B. thailandensis, 5 B. thailandensis-like species, 44 B. ubonensis, and other 25 Burkholderia strains (Tables 1 and Additional file 1: Table S1). Species identification was made on the basis of $\operatorname{rec} A$ and $16 \mathrm{~S}$ rRNA sequences $[17,18]$. B. pseudomallei strains K96243, 576, MSHR840, and MSHR1655 were used as references for the $\mathrm{O}$-antigen types $\mathrm{A}, \mathrm{B}, \mathrm{B} 2$, and rough, respectively [11]. All strains were grown on Luria-Bertani (LB) agar (Difco, USA) for DNA and LPS extractions. DNA was extracted using the Wizard Genomic DNA Purification Kit (Promega, Madison, WI, USA), according to the manufacturer's instructions. LPS was extracted using wholecell lysis according to a previous method [11,20] and separated by SDS-PAGE (Invitrogen, USA).

\section{PCR analysis}

Strains were genotyped for $B$. pseudomallei O-antigen types via multiplex-SYBR-Green real-time PCR in accordance with as previously reported [11]. As the previously published sequences did not detect all nearneighbors expressing type $\mathrm{A}$, this primer pair was redesigned. The new type A primer pair spans the intergenic space of $w b i D$ and $w b i E$ and the primer sequences are: LPSA_For, 5'-ACGGGATCGTACAGTTTCGGATGCT-3'; and LPSA_ Rev, 5'-GAAGATCGTCGCTCGGAGAAT CGT-3'.

\section{Silver staining and serology}

Silver staining was first used to validate $B$. pseudomallei O-antigen type presence in near-neighbor strains, following the previously determined criteria for identification $[11,20]$. Samples were then screened for serocrossreactivity using sera from two Australian melioidosis patients, one serum sample per immunoblot analysis. One patient was infected by B. pseudomallei MSHR1328 expressing type A O-antigen, while another patient was infected by strain MSHR1079 which expressed type B O-antigen [11]. The same samples were also tested serologically using the commercially available monoclonal antibody (mAb) 3D11 (Fitzgerald Industries International Inc., USA), specific to B. mallei LPS [23]. Additionally, LPS samples from all B. thailandensis strains were also tested using mAb Pp-PS-W [13] which is specific to $B$. pseudomallei type A O-polysaccharide (O-PS).

\section{Serum-sensitivity testing}

The susceptibility of the near-neighbor strains to $30 \%$ normal human serum (NHS; Lonza Group LtD., USA) was tested according to a previous method [11,23]. Briefly, strains were grown at $37^{\circ} \mathrm{C}$ overnight with shaking in LB broth and cell concentrations were equilibrated. A 1:1,000 dilution of culture was created in TSBDC (Trypticase soy broth dialysate -treated with Chelex-100) media [32], and grown for five hours. A 1:6:3 vol. ratio of the culture: TSB-DC media:undiluted NHS was incubated for two hours at $37^{\circ} \mathrm{C}$ with no shaking. Total bacterial plate counting was performed on these cultures. E. coli HB101 was used as a negative control.

\section{Whole genome sequencing and genomic analysis}

Whole genome sequencing was performed using 454 sequencing technology (Roche, USA) by the US Army Edgewood Chemical Biological Center (ECBC), Aberdeen, MD. O-antigen biosynthesis gene cluster annotations were made in comparison to the aforementioned reference $B$. pseudomallei types using the BLAST program and Artemis Comparison Tool (ACT) [33]. Annotated Oantigen gene sequences of $B$. mallei strains India 86-5672, KC237, NCTC120; B. thailandensis strains MSMB59, MSMB60, 82172; B. thailandensis-like species strains MSMB121, MSMB12; B. ubonensis strain MSMB57; and unidentified Burkholderia sp. strain MSMB175, were assigned GenBank accessions: JN581990, JN581991, JN581992, JN581997, JN581998, JQ783347, HQ908420, JF745809, JF745807, and JF745808, respectively.

\section{Additional files}

Additional file 1: Table S1. List of Burkholderia strains used in this study, and their identified genotypes and phenotypes.

Additional file 2: Figure S1. SDS-PAGE and immunoblotting analyses of 3 reference LPS banding patterns A, B, and B2 in B. pseudomallei strains K96243 (lane 1), 576 (lane 2), and MSHR840 (lane 3), respectively. Panel A is the silver stained SDS-PAGE. Panels B and C are the immunoblots of LPS samples in panel $A$ which were hybridized against sera from serotype $A$ and $B$ patients, respectively. Lane 4 is the LPS from B. pseudomallei strain MSHR1655 which is rough type and not seroreactive. Lane $L$ is a standard protein ladder.

Additional file 3: Figure S2. Comparison of type A O-antigen biosynthesis clusters. Type A O-antigen is found in four species, from top to bottom, B. oklahomensis, B. pseudomallei, B. mallei, and B. thailandensis. Red indicates nucleotide homology of 78-100\%. The glycosyltransferase gene wbiE (BoklE_010100014785) is truncated in B. oklahomensis E0147 but 
maintains functional. Conversely, insertion of a thymine into the methyltransferase wbiD relative to $B$. pseudomallei $\mathrm{K} 96243$ removes the functionality of this enzyme in E0147, removing it from the comparison.

\section{Competing interests}

Authors declare that they have no competing interests.

\section{Authors' contributions}

AT, BJC and PK conceived of the study. JKS performed major experimental analyses and drafted the manuscript. MM, SAG, JLG, CJA, AD, SG, and MK provided technical assistances. HSG, SMB, MAK, JMI, KSH, and LAM sequenced all Burkholderia genomes used in this study. PK, BJC, and AT reviewed and edited the manuscript. All authors read and approved the final manuscript.

\section{Acknowledgements}

This work was funded by the US Department of Homeland Security contract no. HSHQDC-10-C-00135 to AT.

\section{Author details}

${ }^{1}$ Center for Microbial Genetics and Genomics, Northern Arizona University, Flagstaff, AZ, USA. ${ }^{2}$ Menzies School of Health Research, Darwin, NT, Australia. ${ }^{3}$ BioSciences Division, Edgewood Chemical Biological Center, Aberdeen Proving Ground, MD, USA.

Received: 28 July 2012 Accepted: 31 October 2012 Published: 5 November 2012

\section{References}

1. Raetz CRH, Whitfield C: Lipopolysaccharide endotoxins. Annu Rev Biochem 2002, 71:635-700.

2. Caroff M, Karibian D: Structure of bacterial lipopolysaccharides. Carbohydr Res 2003, 338(23):2431-2447.

3. Alexander C, Rietschel ET: Invited review: bacterial lipopolysaccharides and innate immunity. J Endotoxin Res 2001, 7(3):167-202.

4. Novem V, Shui G, Wang D, Bendt AK, Sim SH, Liu Y, Thong TW, Sivalingam SP, Ooi EE, Wenk MR, et al: Structural and biological diversity of lipopolysaccharides from Burkholderia pseudomallei and Burkholderia thailandensis. Clin Vaccine Immunol 2009, 16(10):1420-1428.

5. Cheng AC, Currie BJ: Melioidosis: epidemiology, pathophysiology, and management. Clin Microbiol Rev 2005, 18(2):383-416.

6. Rotz LD, Khan AS, Lillibridge SR, Ostroff SM, Hughes JM: Public health assessment of potential biological terrorism agents. Emerg Infect Dis 2002, 8(2):225-230.

7. Brett P, Woods D: Structural and immunological characterization of Burkholderia pseudomallei O-polysaccharide-flagellin protein conjugates. Infect Immun 1996, 64(7):2824-2828.

8. Jones SM, Ellis JF, Russell P, Griffin KF, Oyston PCF: Passive protection against Burkholderia pseudomallei infection in mice by monoclonal antibodies against capsular polysaccharide, lipopolysaccharide or proteins. J Med Microbiol 2002, 51(12):1055-1062.

9. Nelson M, Prior JL, Lever MS, Jones HE, Atkins TP, Titball RW: Evaluation of lipopolysaccharide and capsular polysaccharide as subunit vaccines against experimental melioidosis. J Med Microbiol 2004, 53(12):1177-1182.

10. Ngugi SA, Ventura W, Qazi O, Harding SV, Kitto GB, Estes DM, Dell A, Titball RW, Atkins TP, Brown KA, et al: Lipopolysaccharide from Burkholderia thailandensis E264 provides protection in a murine model of melioidosis. Vaccine 2010, 28(47):7551-7555.

11. Tuanyok A, Stone JK, Mayo M, Kaestli M, Gruendike J, Georgia S, Warrington S, Mullins T, Allender CJ, Wagner DM, et al: The genetic and molecular basis of O-antigenic diversity in Burkholderia pseudomallei lipopolysaccharide. PLoS Negl Trop Dis 2012, 6(1):e1453.

12. Brett PJ, DeShazer D, Woods DE: Burkholderia thailandensis sp. nov., a Burkholderia pseudomallei-like species. Int J Syst Bacteriol 1998, 48(1):317-320.

13. Burtnick MN, Brett PJ, Woods DE: Molecular and physical characterization of Burkholderia mallei O Antigens. J Bacteriol 2002, 184(3):849-852.

14. Brett PJ, Burtnick MN, Heiss C, Azadi P, DeShazer D, Woods DE, Gherardini FC: Burkholderia thailandensis oacA mutants facilitate the expression of Burkholderia mallei-Like O Polysaccharides. Infect Immun 2011, 79(2):961-969.
15. Knirel YA, Paramonov NA, Shashkov AS, Kochetkov NK, Yarullin RG, Farber SM, Efremenko VI: Structure of the polysaccharide chains of Pseudomonas pseudomallei lipopolysaccharides. Carbohydr Res 1992, 233:185-193.

16. Perry M, MacLean L, Schollaardt T, Bryan L, Ho M: Structural characterization of the lipopolysaccharide $\mathrm{O}$ antigens of Burkholderia pseudomallei. Infect Immun 1995, 63(9):3348-3352.

17. Gee JE, Glass MB, Novak RT, Gal D, Mayo MJ, Steigerwalt AG, Wilkins PP, Currie BJ: Recovery of a Burkholderia thailandensis-like isolate from an Australian water source. BMC Microbiol 2008, 8:54.

18. Godoy D, Randle G, Simpson AJ, Aanensen DM, Pitt TL, Kinoshita R, Spratt BG: Multilocus sequence typing and evolutionary relationships among the causative agents of melioidosis and glanders, Burkholderia pseudomallei and Burkholderia mallei. J Clin Microbiol 2003, 41(5):2068-2079.

19. Glass MB, Steigerwalt AG, Jordan JG, Wilkins PP, Gee JE: Burkholderia oklahomensis sp. nov., a Burkholderia pseudomallei-like species formerly known as the Oklahoma strain of Pseudomonas pseudomallei. Int J Syst Evol Microbiol 2006, 56(9):2171-2176.

20. Woods DE, Jeddeloh JA, Fritz DL, DeShazer D: Burkholderia thailandensis E125 harbors a temperate bacteriophage specific for Burkholderia mallei. J Bacteriol 2002, 184(14):4003-4017.

21. Tuanyok A, Leadem BR, Auerbach RK, Beckstrom-Sternberg SM, BeckstromSternberg JS, Mayo M, Wuthiekanun V, Brettin TS, Nierman WC, Peacock SJ, et al: Genomic islands from five strains of Burkholderia pseudomallei. BMC Genomics 2008, 9:566.

22. Brett PJ, Burtnick MN, Woods DE: The wbiA locus is required for the 2-Oacetylation of lipopolysaccharides expressed by Burkholderia pseudomallei and Burkholderia thailandensis. FEMS Microbiol Lett 2003, 218(2):323-328.

23. DeShazer D, Brett PJ, Woods DE: The type II O-antigenic polysaccharide moiety of Burkholderia pseudomallei lipopolysaccharide is required for serum resistance and virulence. Mol Microbiol 1998, 30(5):1081-1100.

24. Levy A, Merritt AJ, Aravena-Roman M, Hodge MM, Inglis TJJ: Expanded Range of Burkholderia Species in Australia. AmJTrop Med Hyg 2008 78(4):599-604

25. Marshall K, Shakya S, Greenhill AR, Padilla G, Baker A, Warner JM: Antibiosis of Burkholderia ubonensis against Burkholderia pseudomallei, the causative agent for melioidosis. Southeast Asian J Trop Med Public Health 2010, 41(4):904-912.

26. Sim BMQ, Chantratita N, Ooi WF, Nandi T, Tewhey R, Wuthiekanun V, Thaipadungpanit J, Tumapa S, Ariyaratne P, Sung W-K, et al: Genomic acquisition of a capsular polysaccharide virulence cluster by nonpathogenic Burkholderia isolates. Genome Biol 2010, 11(8):R89.

27. Kanaphun $P$, Thirawattanasuk N, Suputtamongkol $Y$, Naigowit $P$, Dance DAB, Smith MD, White NJ: Serology and carriage of pseudomonas pseudomallei: a prospective study in 1000 hospitalized children in Northeast Thailand. J Infect Dis 1993, 167(1):230-233.

28. Smith M, Angus B, Wuthiekanun V, White N: Arabinose assimilation defines a nonvirulent biotype of Burkholderia pseudomallei. Infect Immun 1997 65(10):4319-4321.

29. Harris PNA, Ketheesan N, Owens L, Norton RE: Clinical features that affect indirect-hemagglutination-assay responses to Burkholderia pseudomallei. Clin Vaccine Immuno/ 2009, 16(6):924-930.

30. Ashdown LR, Guard RW: The prevalence of human melioidosis in Northern Queensland. AmJTrop Med Hyg 1984, 33(3):474-478.

31. Lazzaroni SM, Barnes JL, Williams NL, Govan BL, Norton RE, LaBrooy JT, Ketheesan N: Seropositivity to Burkholderia pseudomallei does not reflect the development of cell-mediated immunity. Trans $R$ Soc Trop Med Hyg 2008, 102(Supplement 1):S66-S70.

32. Ohman DE, Sadoff JC, Iglewski BH: Toxin A-deficient mutants of Pseudomonas aeruginosa PA103: isolation and characterization. Infect Immun 1980, 28(3):899-908.

33. Carver TJ, Rutherford KM, Berriman M, Rajandream MA, Barrell BG, Parkhill ACT: the artemis comparison tool. Bioinformatics 2005, 21(16):3422-3423.

\section{doi:10.1186/1471-2180-12-250}

Cite this article as: Stone et al:: Detection of Burkholderia pseudomallei O-antigen serotypes in near-neighbor species. BMC Microbiology 2012 12:250. 